\title{
Analytical approximations of diving-wave imaging in constant-gradient medium
}

\author{
Alexey Stovas ${ }^{1}$ and Tariq Alkhalifah ${ }^{2}$
}

\begin{abstract}
Full-waveform inversion (FWI) in practical applications is currently used to invert the direct arrivals (diving waves, no reflections) using relatively long offsets. This is driven mainly by the high nonlinearity introduced to the inversion problem when reflection data are included, which in some cases require extremely low frequency for convergence. However, analytical insights into diving waves have lagged behind this sudden interest. We use analytical formulas that describe the diving wave's behavior and traveltime in a constant-gradient medium to develop insights into the traveltime moveout of diving waves and the image (model) point dispersal (residual) when the wrong velocity is used. The explicit formulations that describe these phenomena reveal the high dependence of diving-wave imaging on the gradient and the initial velocity. The analytical image point residual equation can be further used to scan for the best-fit linear velocity model, which is now becoming a common sight as an initial velocity model for FWI. We determined the accuracy and versatility of these analytical formulas through numerical tests.
\end{abstract}

\section{INTRODUCTION}

Recently, direct arrivals and specifically diving waves are experiencing a new lease on life as one of the major sources of velocity information in full-waveform inversion (FWI) (Pratt et al., 1996; Virieux and Operto, 2009; Plessix and Cao, 2011). Those inverted velocities from diving waves tend to have low wavelength characteristics reflecting the smooth nature of its dependency on velocity perturbations, which is mostly embedded in the kinematic aspects of the diving waves. Also in many cases, these inverted models provide the background medium needed to start a reflection-based FWI inversion if low enough frequencies are available in the data. In fact, inverted models from pure diving waves, when used for imaging, are capable of producing a focused image of the subsurface (Plessix and Rynja, 2010). This is in spite of the fact that diving waves did not experience any reflections. They are, however, sensitive to the same medium velocities. In fact, for imaging, diving waves actually tend to share the same velocity wavelength spectrum necessary to focus an image or flatten an angle gather. The higher model wavenumbers attained from a reflection-based FWI are usually responsible for increasing the resolution of the image, but it usually adds little to the focusing. The large offsets and low frequencies acquired nowadays allow us to record diving waves that penetrate to large depths, depths usually reserved for reflected data.

In a general waveform inversion, we rarely distinguish between diving waves and reflections and the update to the velocity model usually holds information from both. Recently, attempts to split the two have gained considerable attention (Tang et al., 2013) because they tend to provide very different model update behavior (Sirgue and Pratt, 2004). In the hierarchal scheme of FWI, in which we tend to want to update the background velocity first (using low frequencies and early arrivals) until we approach an acceptable model for reflections, our inversion is focused on diving waves to get that acceptable model.

The reflection moveout behavior and its focusing after imaging have been studied for years, in which time we have gained considerable analytical insights into the behavior of reflections. Understandably, similar studies for diving waves have lagged behind because our focus was reflections to the point that our field was referred to as reflection seismology. We could still find interesting articles that discuss diving waves and specifically its traveltime and acquisition features, such as that of Levin (1996). The importance of diving waves is now being realized. Shen (2013) develops an approach to measure the defocusing in imaging diving waves in the subsurface when the wrong velocity is used. This defocusing (image

\footnotetext{
Manuscript received by the Editor 22 October 2013; revised manuscript received 28 March 2014; published online 24 June 2014.

${ }^{1}$ Norwegian University of Science and Technology, Trondheim, Norway. E-mail: alexey.stovas@ntnu.no.

${ }^{2}$ King Abdullah University of Science and Technology (KAUST), Astronomy and Geophysical Research Institute, Thuwal, Saudi Arabia. E-mail: tariq. alkhalifah@kaust.edu.sa.

(C) 2014 Society of Exploration Geophysicists. All rights reserved.
} 
point shift from zero offset) can then be used to update the velocity model. The update kernel is essentially similar to that experienced for FWI of diving waves.

In this paper, we develop analytical formulations to describe the traveltime moveout for diving waves in a constant-gradient medium. The combination of the forward and backward mapping formulas provides us with analytical description of the image point (or model point) dispersal (or defocusing) when the wrong velocity or gradient is used. We test these approximate formulations by comparing them with the misfocusing we obtain from diving waves using reverse time migration (RTM).

\section{DIVING-WAVE IMAGE MOVEOUT}

For a constant-gradient velocity model

$$
v(z)=v_{0}+G z
$$

the ray trajectory for diving waves with a fixed value of horizontal slowness $p$ is given by an arc of a circle (Figure 1, Slotnick, 1959) with a radius $R=\frac{1}{p G}$, where $G$ is the gradient of the velocity and $v_{0}$ is the velocity on the model top (the earth surface), and the position of the center given by $X_{0}=\frac{\sqrt{1-p^{2} v_{0}^{2}}}{p G}$ and $Z_{0}=-\frac{v_{0}}{G}$, as

$$
\left(z+\frac{v_{0}}{G}\right)^{2}+\left(x-\frac{\sqrt{1-p^{2} v_{0}^{2}}}{p G}\right)^{2}=\frac{1}{p^{2} G^{2}} .
$$

The position of the turning point (Figure 1) is defined as

$$
x_{0}=\frac{\sqrt{1-p^{2} v_{0}^{2}}}{p G}, \quad z_{0}=\frac{1-p v_{0}}{p G},
$$

with traveltime from $(0,0)$ to $\left(x_{0}, z_{0}\right)$ given by

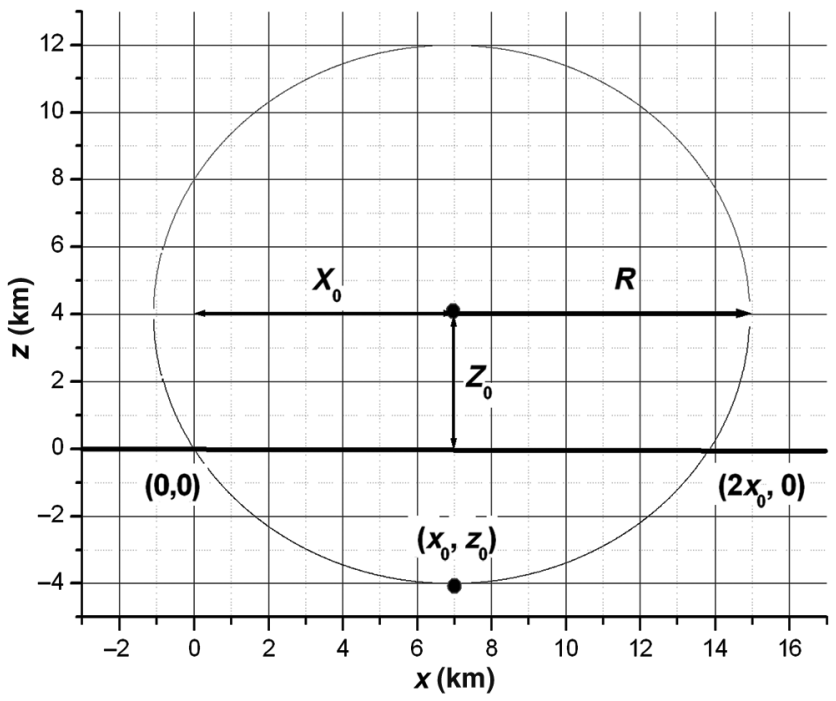

Figure 1. The diving-wave ray trajectory.

$$
t_{0}=\frac{1}{G} \log \left[\frac{1+\sqrt{1-p^{2} v_{0}^{2}}}{p v_{0}}\right]
$$

The point $\left(x_{0}, z_{0}\right)$ is the image point when the correct velocity model is used for migration.

\section{Perturbation in velocity gradient}

With a change in gradient only, the center of the circle moves along the radius (Figure 2) according to the following relation:

$$
z(x)=-\frac{p v_{0} x}{\sqrt{1-p^{2} v_{0}^{2}}}
$$

Assuming perturbation $\Delta G$ in gradient, $v_{n}(z)=v_{0}+(G+$ $\Delta G) z$, we trace the ray with the same ray parameter $p$ (Figure 3). The parameters for a turning point in a perturbed medium can easily be obtained from equations 3 and 4 :

$$
\begin{aligned}
& x_{0 n}=\frac{\sqrt{1-p^{2} v_{0}^{2}}}{p(G+\Delta G)}, \\
& z_{0 n}=\frac{1-p v_{0}}{p(G+\Delta G)}, \\
& t_{0 n}=\frac{1}{(G+\Delta G)} \log \left[\frac{1+\sqrt{1-p^{2} v_{0}^{2}}}{p v_{0}}\right] .
\end{aligned}
$$

Now, we define the point $P\left(x_{P}, z_{P}\right)$ on the ray in the perturbed medium that possess the same ray parameter $p$, and the traveltime from the origin to this point is equal to $t_{0}$ computed for the unperturbed medium (equation 4).

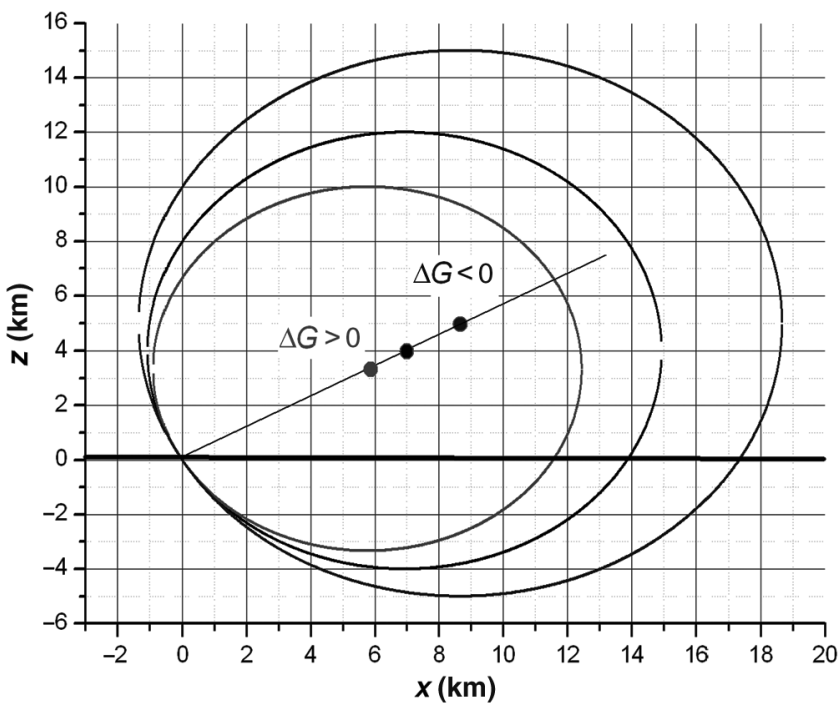

Figure 2. Change in ray trajectory due to change in velocity gradient. 
The traveltime to point $P\left(x_{P}, z_{P}\right)$ located at the right side leg of the ray is given by

$$
\begin{aligned}
t_{P}= & 2 t_{0 n}-t\left(p, z_{P}\right) \\
= & \frac{1}{(G+\Delta G)}\left(2 \log \left(\frac{1+\sqrt{1-p^{2} v_{0}^{2}}}{p v_{0}}\right)\right. \\
& \left.-\log \left(\frac{v_{n}\left(z_{P}\right)}{v_{0}} \frac{1+\sqrt{1-p^{2} v_{0}^{2}}}{1+\sqrt{1-p^{2} v_{n}^{2}\left(z_{P}\right)}}\right)\right),
\end{aligned}
$$

where $t\left(p, z_{P}\right)$ is the traveltime between points $P$ and $\left(2 x_{n 0}, 0\right)$ (see Appendix A).

The lateral position of this point $x_{P}$ is defined by

$$
x_{P}=2 x_{0 n}-x\left(p, z_{P}\right)=\frac{\sqrt{1-p^{2} v_{0}^{2}}+\sqrt{1-p^{2} v_{n}^{2}\left(z_{P}\right)}}{p(G+\Delta G)} \text {, }
$$

where $x\left(p, z_{P}\right)$ is the lateral distance between the points $P$ and $\left(2 x_{n 0}, 0\right)$ (see Appendix A).

By setting the condition that traveltime $t_{P}$ (from equation 7 ) is equal to traveltime $t_{0}$ (from equation 4 ),

$$
t_{P}=t_{0}
$$

we can find the depth $z_{P}$ and lateral position $x_{P}$ that depend on the ray parameter $p$,

$$
\begin{gathered}
z_{P}=\frac{\frac{2\left(p v_{0}\right)^{\frac{\Delta G}{G}}\left(1+\sqrt{1-p^{2} v_{0}^{2}}\right)^{\frac{\Delta G}{G}}}{p\left(G v_{0}\right)^{\frac{2 \Delta G}{G}}+\left(1+\sqrt{1-p^{2} v_{0}^{2}}\right)^{\frac{2 \Delta G}{G}}}-p v_{0}}{p(G+\Delta G)}, \\
x_{P}=\frac{\left(p v_{0}\right)^{\frac{2 \Delta G}{G}}+\left(1+\sqrt{1-p^{2} v_{0}^{2}}\right)^{\frac{2 \Delta G}{G}}+\sqrt{1-p^{2} v_{0}^{2}}}{p(G+\Delta G)} .
\end{gathered}
$$

It is convenient to introduce the traveltime instead of the ray parameter by solving equation $4 t_{0}=T$, where $T$ is the variable corresponding to half of recorded traveltime:

$$
p=\frac{1}{v_{0}} \frac{2 e^{G T}}{e^{2 G T}+1}
$$

Substituting equation 11 into equations 10 gives

$$
\begin{aligned}
& z_{P}=\frac{v_{0}}{(G+\Delta G)} \frac{\left(e^{G T} e^{\Delta G T}-1\right)\left(e^{G T}-e^{\Delta G T}\right)}{e^{G T}\left(e^{2 \Delta G T}+1\right)}, \\
& x_{P}=\frac{v_{0}}{(G+\Delta G)} \frac{\left(e^{2 G T} e^{2 \Delta G T}-1\right)}{e^{G T}\left(e^{2 \Delta G T}+1\right)} .
\end{aligned}
$$

The parametric equations 12 give exact imaging point dispersal due to the incorrect velocity gradient $G+\Delta G$. By setting $\Delta G=0$, we obtain from equation 12

$$
\begin{aligned}
& z_{P}(\Delta G=0)=\frac{v_{0}}{G} \frac{\left(e^{G T}-1\right)^{2}}{2 e^{G T}}=z_{0} \\
& x_{P}(\Delta G=0)=\frac{v_{0}}{G} \frac{\left(e^{2 G T}-1\right)}{2 e^{G T}}=x_{0} .
\end{aligned}
$$

Now, we compose the dependence of the image point dispersal $\Delta x\left(z_{P}\right)$, where $\Delta x=x_{P}-x_{0}$. The function $\Delta x\left(z_{P}\right)$ gives the moveout curve in the image gather.

The perturbation in the gradient gives a limited series:

$$
\begin{aligned}
& z_{P}=z_{0}\left(1-\frac{\Delta G}{G}\right) \\
& \Delta x=\frac{v_{0}}{G} \frac{\left(1-e^{2 G T}+G T\left(1+e^{2 G T}\right)\right)}{2 e^{G T}} \frac{\Delta G}{G}\left(1-\frac{\Delta G}{G}\right) .
\end{aligned}
$$

From equations 14 , one can evaluate the position of the focusing point with respect to the correct point.

From parametric equations 12, we can compose the series

$$
\Delta x^{2}=a_{3} z_{P}^{3}+a_{4} z_{P}^{4}+a_{5} z_{P}^{5}+\ldots,
$$

where the coefficients are given by (see Appendix B)

$$
\begin{aligned}
& a_{3}=\frac{8}{9} \frac{\Delta G^{2}}{v_{0}(G-\Delta G)}, \\
& a_{4}=-\frac{4}{45} \frac{\Delta G^{2}\left(G^{2}-9 \Delta G^{2}\right)}{v_{0}^{2}(G-\Delta G)^{2}}, \\
& a_{5}=\frac{2}{4725} \frac{\Delta G^{2}\left(39 G^{4}-542 G^{2} \Delta G^{2}+1719 \Delta G^{4}\right)}{v_{0}^{3}(G-\Delta G)^{3}} .
\end{aligned}
$$

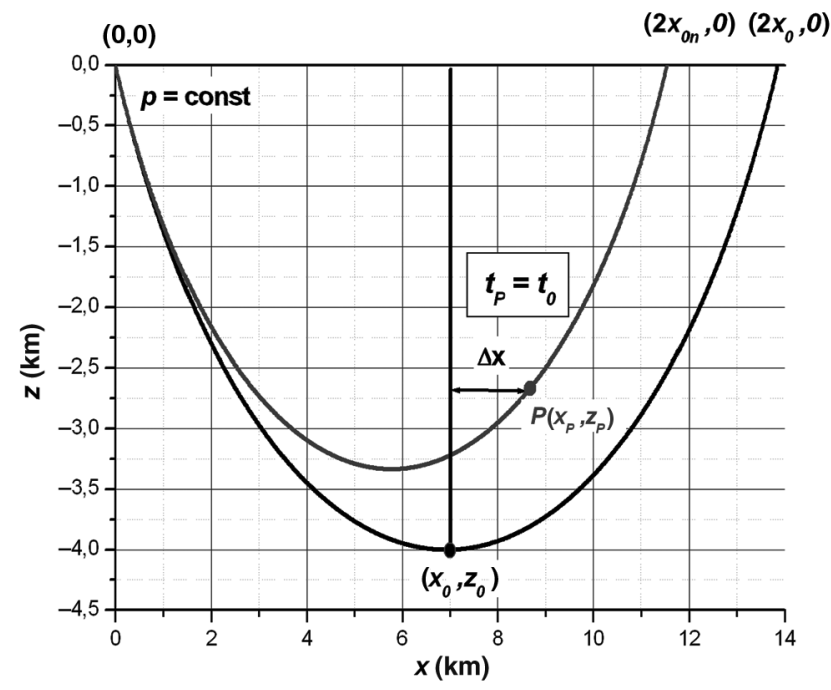

Figure 3. Position of the focusing point in the medium with perturbed velocity gradient. 
From series 15 with coefficients 16 , we can define the coefficients in the Shanks transform to improve the convergence of series 15 and define the approximation:

$$
\Delta x=\frac{2}{3} \Delta G z_{P} \sqrt{\frac{2 z_{P}}{(G-\Delta G) v_{0}} \frac{1+\frac{\left(9 G^{2}-\Delta G^{2}\right) z_{P}}{105(G-\Delta G) v_{0}}}{1+\frac{\left(39 G^{2}-191 \Delta G^{2}\right) z_{P}}{210(G-\Delta G) v_{0}}}} .
$$

Here, we assume that the perturbation in gradient is smaller than the correct gradient value; i.e., $G-\Delta G>0$.

\section{Perturbation in zero-depth velocity}

With a change in the zero-depth velocity of only $v_{n}(z)=$ $v_{0}+\Delta v+G z$, the equation for moving of the center position takes the form (Figure 4),

$$
z(x)=-\sqrt{\frac{1}{p^{2} G^{2}}-x^{2}} .
$$

Using the same imaging condition for the focusing point as above leads to following equations:

$$
\begin{aligned}
& z_{P}=\frac{v_{0}}{G} \frac{\sqrt{1-r_{2}^{2}}\left(\sqrt{1-r_{1}^{2}}-1+r_{1} r_{2}\right)}{\sqrt{1-r_{1}^{2}}\left(1-r_{1} r_{2}\right)}, \\
& \Delta x=\frac{v_{0}}{G} \frac{r_{1} r_{2}\left(r_{1}-r_{2}\right)}{\sqrt{1-r_{1}^{2}}\left(1-r_{1} r_{2}\right)},
\end{aligned}
$$

where $r_{1}=\sqrt{1-p^{2} v_{0}^{2}}$ and $r_{2}=\sqrt{1-p^{2}\left(v_{0}+\Delta v\right)^{2}}$.

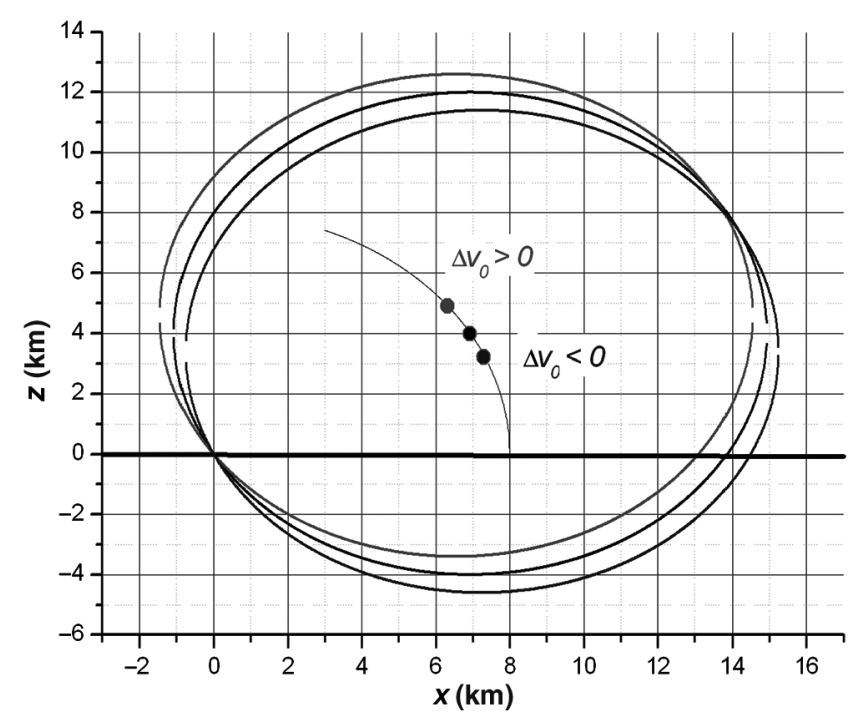

Figure 4. Change in ray trajectory due to change in zero-depth velocity.
If $\Delta v=0, r_{1}=r_{2}$ and

$$
z_{P}=z_{0}=\frac{v_{0}}{G} \frac{\left(1-\sqrt{1-r_{1}^{2}}\right)}{\sqrt{1-r_{1}^{2}}}, \quad \Delta x=0,
$$

with

$$
x_{0}=\frac{v_{0}}{G} \frac{r_{1}}{\sqrt{1-r_{1}^{2}}} .
$$

Perturbation in zero-depth velocity change in equations 19 gives

$$
\begin{aligned}
& z_{P}=z_{0}-\frac{\Delta v}{G}-\frac{\Delta v^{2}}{2 G p v_{0}^{2}\left(1-p^{2} v_{0}^{2}\right)}, \\
& \Delta x=\frac{\Delta v \sqrt{1-p^{2} v_{0}^{2}}}{G p v_{0}}-\frac{\Delta v^{2}}{2 G p v_{0}^{2} \sqrt{1-p^{2} v_{0}^{2}}} .
\end{aligned}
$$

Now, we need to compute the horizontal slowness that gives $z_{P}=0$. It can be computed from equation 19 by solving the following equation:

$$
\sqrt{1-r_{1}^{2}}-1+r_{1} r_{2}=0
$$

The solution of equation 23 depends on the sign of $\Delta v$,

$$
p_{c}=\left\{\begin{array}{cc}
\frac{1}{2 v_{0}}\left(\sqrt{1+\frac{8}{(1+\gamma)^{2}}}-1\right), & \Delta v>0 \\
\frac{1}{v_{0}}, & \Delta v \leq 0
\end{array},\right.
$$

where $\gamma=\Delta v / v_{0}$.

The value of $\Delta x$ at zero depth is defined as

$$
\Delta x(0)=\left\{\begin{array}{cc}
\frac{\left(1-p_{c} v_{0}\right)^{2}}{G p_{c} \sqrt{1-p_{c}^{2} v_{0}^{2}},} & \Delta v>0 \\
0, & \Delta v \leq 0
\end{array},\right.
$$

being zero for $\Delta v \leq 0$ and being a positive number for $\Delta v>0$. For a small positive $\gamma$, we can evaluate $\Delta x(0)$ as

$$
\Delta x(0) \approx \frac{4 v_{0}}{3 G} \sqrt{\frac{2}{3}} \gamma^{3 / 2} .
$$

The limited series for $\Delta x\left(z_{P}\right)$ is different from the one obtained for the medium perturbed with velocity gradient and is given by

$$
\Delta x(z)=\Delta x(0)+a_{1} z_{P}+a_{2} z_{P}^{2},
$$

with coefficients (see Appendix B) 


$$
\begin{aligned}
& a_{1}=\frac{\sqrt{1-p_{c}^{2} v_{0}^{2}}-\sqrt{1-p_{c}^{2} v_{0}^{2}(1+\gamma)^{2}}}{p_{c}^{2} v_{0}^{2}(1+\gamma)}, \\
& a_{2}=\frac{G\left(\sqrt{1-p_{c}^{2} v_{0}^{2}}-\sqrt{1-p_{c}^{2} v_{0}^{2}(1+\gamma)^{2}}\right)\left(1-\sqrt{\left(1-p_{c}^{2} v_{0}^{2}\right)\left(1-p_{c}^{2} v_{0}^{2}(1+\gamma)^{2}\right)}\right)^{3}}{2 v_{0}^{4} p_{c}^{3}(1+\gamma)^{2}\left(\sqrt{\left(1-p_{c}^{2} v_{0}^{2}\right)\left(1-p_{c}^{2} v_{0}^{2}(1+\gamma)^{2}\right)}-1+p_{c}^{4} v_{0}^{4}(1+\gamma)^{2}\right)} .
\end{aligned}
$$

To stabilize the approximation 27 , we compute the infinite depth limit,

$$
a_{\infty}=\lim _{z_{P} \rightarrow \infty}\left(\frac{\Delta x}{z_{P}}\right)=\frac{\gamma(\gamma+2)}{2(\gamma+1)} .
$$

Then we define the rational approximation in the following form:

$$
\Delta x(z)=\Delta x(0)+a_{1} z_{P} \frac{1+c z_{P}}{1+d z_{P}}
$$

where parameters $c$ and $d$ are defined as

$$
\begin{aligned}
& c=\frac{a_{\infty} a_{2}}{a_{1}\left(a_{\infty}-a_{1}\right)}, \\
& d=\frac{a_{2}}{\left(a_{\infty}-a_{1}\right)} .
\end{aligned}
$$

For example, for negative $\gamma$ values, we have

$$
\begin{aligned}
\Delta x(0) & =0, \\
a_{1} & =-\frac{\sqrt{-\gamma(2+\gamma)}}{(1+\gamma)}, \\
a_{2} & =\frac{G}{2 v_{0}(1+\gamma)^{2} \sqrt{-\gamma(2+\gamma)}},
\end{aligned}
$$

and

$$
\begin{aligned}
& c=\frac{G}{2 v_{0}(1+\gamma) \sqrt{-\gamma(2+\gamma)}(2-\sqrt{-\gamma(2+\gamma)})}, \\
& d=-\frac{G}{v_{0}(1+\gamma) \gamma(2+\gamma)(2-\sqrt{-\gamma(2+\gamma)})} .
\end{aligned}
$$

\section{IMAGE POINT DISPERSAL}

The position of the turning point (equation 3) in the shot gather can be described by the shifted hyperbola equation:

$$
\left(z_{0}+\frac{v_{0}}{G}\right)^{2}=x_{0}^{2}+\frac{v_{0}^{2}}{G^{2}},
$$

whereas the dispersal of the image point depends both on error in the gradient (or the zero-depth velocity) and depth (Figure 5). One can see that the larger the depth, the larger is the dispersal of the image point due to the incorrect velocity model.

\section{NUMERICAL TESTS}

To test the moveout approximations, we select the constantgradient velocity model with the parameters $v_{0}=2 \mathrm{~km} / \mathrm{s}$ and $G=0.5 \mathrm{~s}^{-1}$. The moveout curves on the image gather after migration with incorrect velocity gradient $\Delta G= \pm 0.1 \mathrm{~s}^{-1}$ are shown in Figure 6. Notice that the curves corresponding to errors with different signs are not symmetric. An accuracy test of the moveout

a)

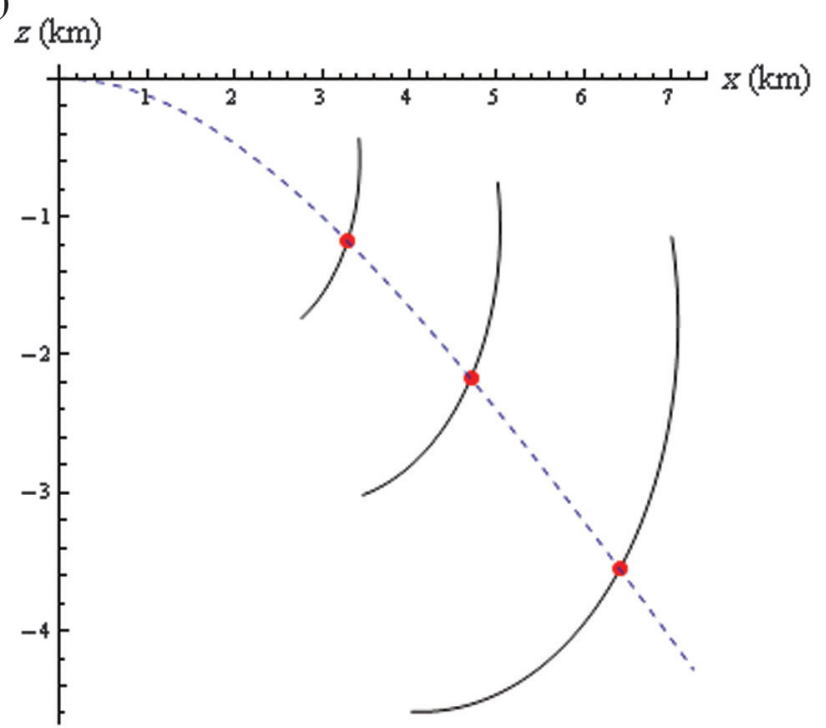

b)

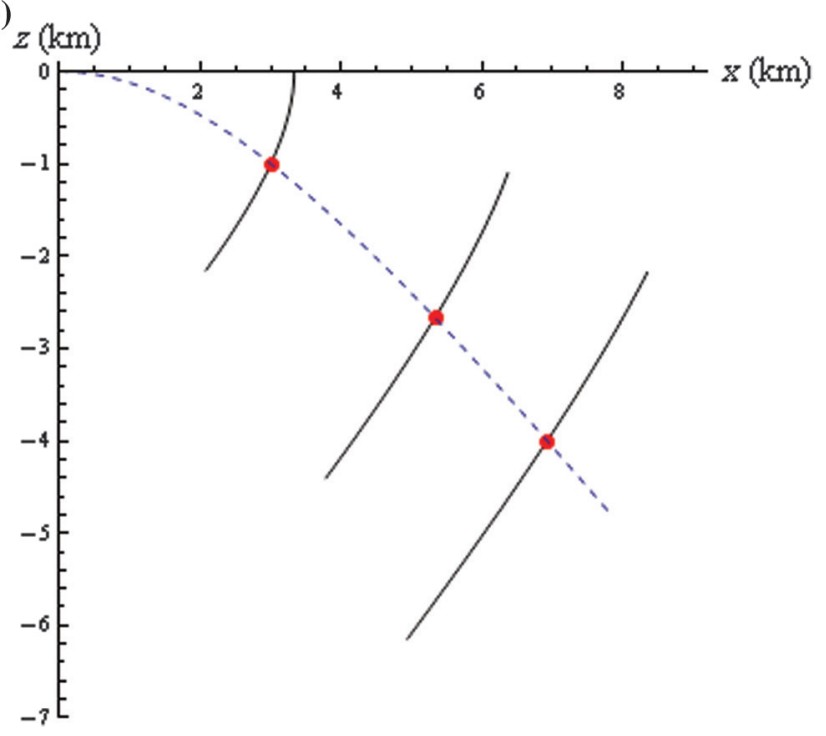

Figure 5. Focusing point dispersal with depth due to (a) the gradient change and (b) the zero-depth velocity change. The exact model values are $v_{0}=2 \mathrm{~km} / \mathrm{s}$ and $G=0.5 \mathrm{~s}^{-1}$. The gradient error range is $\Delta G=[-0.3,0.3] \mathrm{s}^{-1}$, and the zero-depth velocity error is $\Delta v=[-0.5,0.5] \mathrm{km} / \mathrm{s}$. The position of the turning point in the exact model is shown by the dashed line. 
approximations, given by the limited series 15 and the Shanks transform 17, is illustrated in Figure 7. One can see that the Shanks transform significantly improves the accuracy of moveout approximation.

The graph for $\Delta x(0)$ versus $\gamma$ from incorrect zero-depth velocity experiment is shown in Figure 8. It is computed from combining equations 24 and 25 . One can see that $\Delta x(0)$ is increasing with $\Delta v$. The moveout curves due to incorrect zero-depth velocity, $\Delta v=$ $\pm 0.2 \mathrm{~km} / \mathrm{s}$ and the results from moveout approximations from the limited series 27 and rational approximation 30 are shown in Figure 9. Notice that adding the second-order term in the limited series (blue line) does not improve the approximation because it breaks down very quickly, especially for negative $\Delta v$. On the contrary, the rational approximation performs very well both for positive and negative $\Delta v$.

\section{COMPARISON WITH RTM}

Using a linear velocity model, we reproduce the example shown in Shen (2013), in which the initial velocity is set to $2 \mathrm{~km} / \mathrm{s}$ and modeling is performed with a gradient of $3 \mathrm{~s}^{-1}$. The resulting common-shot gather is shown in Figure 10. It replicates many of the synthetic data typically obtained with an initial velocity model for FWI.

Imaging the shot gather using RTM with a space lag imaging condition allows us to obtain common-image point gathers. Figure 11 shows the common-image gathers from applying RTM

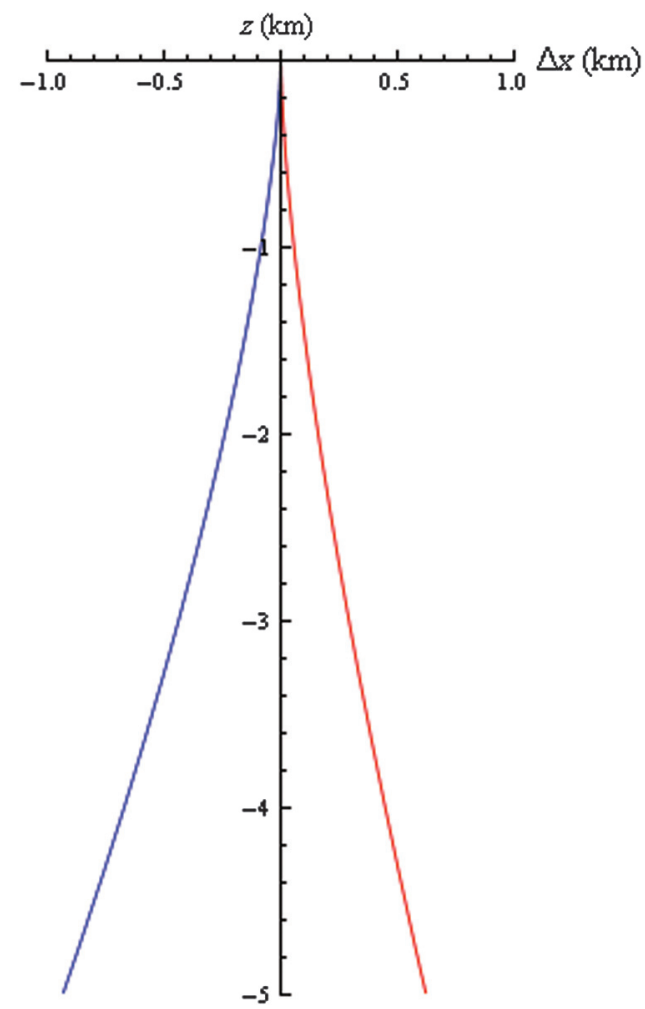

Figure 6. The $\Delta x\left(z_{P}\right)$ for perturbation in velocity gradient of different signs $(\Delta G>0$, red line; $\Delta G<0$, blue line). The parameters are $v_{0}=2 \mathrm{~km} / \mathrm{s}, G=0.5 \mathrm{~s}^{-1}$, and $\Delta G= \pm 0.1 \mathrm{~s}^{-1}$.
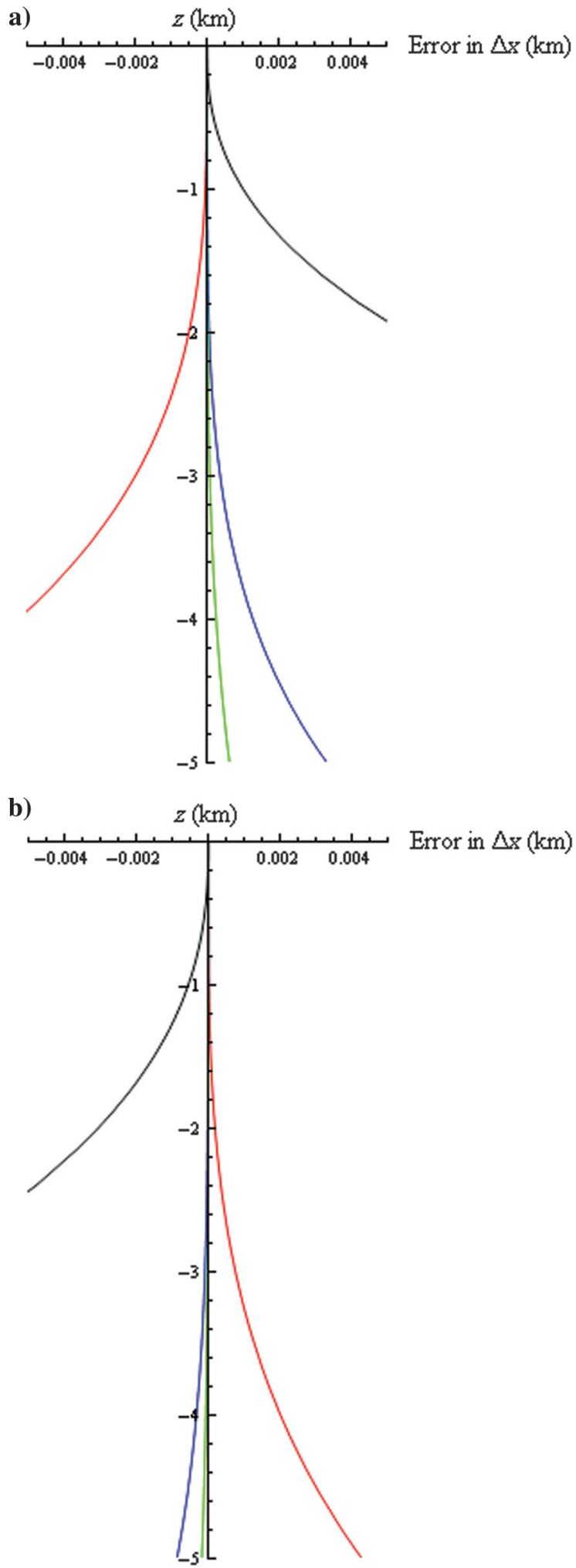

Figure 7. The accuracy of the limited series approximation 15 with different number of terms (one term, black line; two terms, red line; and three terms, blue line) and Shanks transform from equation 17 (green line). The case with $\Delta G>0$ is shown to the left, and $\Delta G<0$ is shown to the right. 
on the synthetic data shown in Figure 10 using the accurate velocity model with a gradient $G=3 \mathrm{~s}^{-1}$ (Figure 11a), using an inaccurate gradient $G=2.5 \mathrm{~s}^{-1}$ (Figure 11b), and using an inaccurate gradient $G=2 \mathrm{~s}^{-1}$ (Figure 11c). We overlay the residual curve predicted by equation 17. Despite the approximate nature of this equation, it predicted with reasonable accuracy these residuals in spite of the large error in gradient. Now, let us do a similar test, but we will focus on the influence of having an error in the initial velocity instead of the gradient. Figure 12 illustrates the common-image gathers from applying RTM on the synthetic data shown in Figure 10 using the accurate velocity model with an initial velocity $v_{0}=2 \mathrm{~km} / \mathrm{s}$ (Figure 12a), using an inaccurate initial velocity $v_{0}=1.5 \mathrm{~km} / \mathrm{s}$ (Figure $12 \mathrm{~b}$ ), and using an inaccurate velocity $v_{0}=$ $1 \mathrm{~km} / \mathrm{s}$ (Figure 12c). The moveout has very different characteristics in this case. It tends to bend inward. We overlay the residual curves predicted by equation 30 . One can see that the residual curves predicted by analytical approximations fit very well to the data and can be used to scan for the best velocity model.

\section{DISCUSSION}

The image point dispersal for diving waves is a good indicator of the accuracy of the velocity used to represent the background model. Like diving-wave FWI, it is sensitive mainly to the longwavelength component of the velocity model, and it can be used to invert for such velocities (Shen, 2013). Developing analytical insights into such a relation can help us figure out the sensitivity features of diving waves, which is mostly given by the kinematics of the wavefield (Plessix and Cao, 2011). Such image gathers for diving waves are computable at every lateral space location. As such, considering its extension vertically to the depth penetrated by the diving waves, it reflects the zero-wavenumber nature of its image resolution. However, its dispersal behavior in the common-image representation provides a tool for estimating reasonably high resolution models, as we see from FWI applications using diving waves. The depth of penetration, which is clearly evident in the commonimage gathers, is dependent on the available offsets and the nature of the velocity increase with depth. The approximate formulations provided here reduce the dispersal moveout description to be dependent on two parameters, the surface velocity and the gradient. Regardless of the depth penetration, a moveout scan over these two parameters, or at least the gradient, can yield an approximate

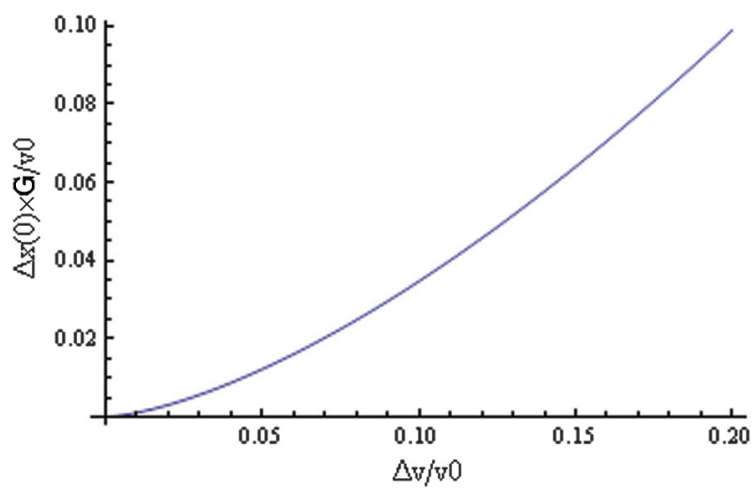

Figure 8 . The value of $G \Delta x_{0} / v_{0}$ versus relative change in zerodepth velocity computed from equation 25 .

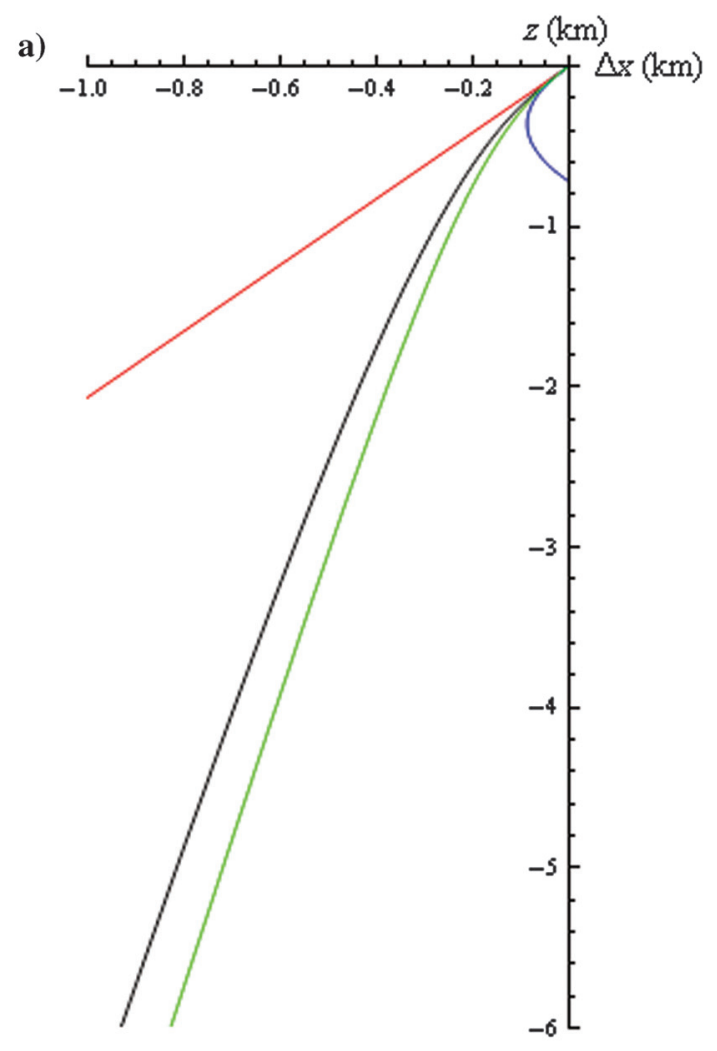

b)

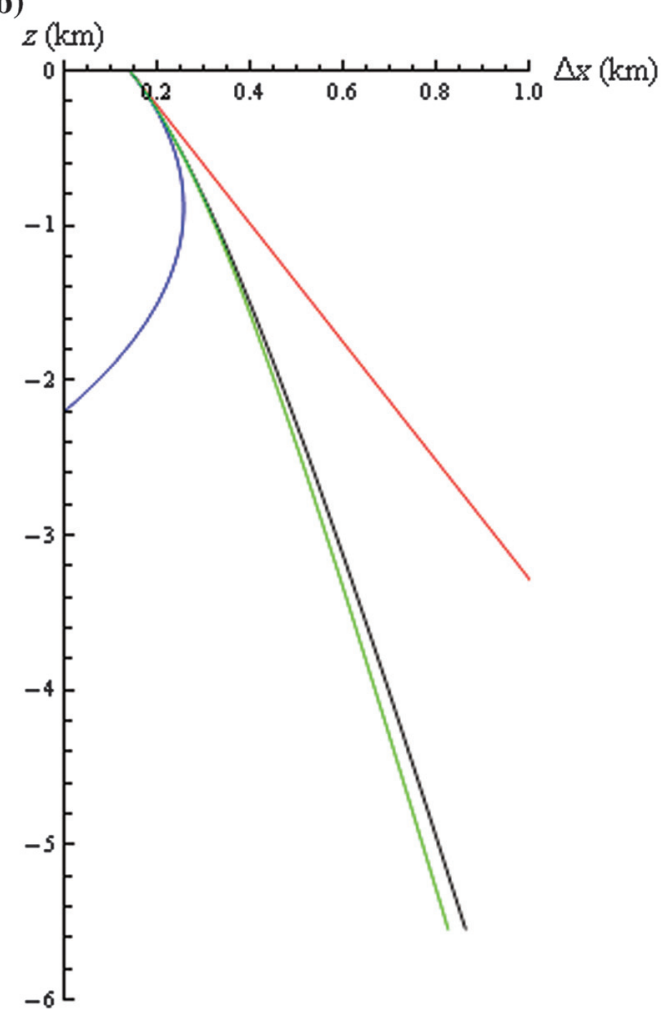

Figure 9. Accuracy of approximations 27 and 30 for (a) negative and (b) positive $\Delta v$. The parameters are $v_{0}=2 \mathrm{~km} / \mathrm{s}, G=0.5 \mathrm{~s}^{-1}$, and $\Delta v= \pm 0.2 \mathrm{~km} / \mathrm{s}$. The exact moveout is shown by the black line, and the two- and three-term limited series are shown with red and blue lines, respectively. The rational approximation is shown by the green line. 


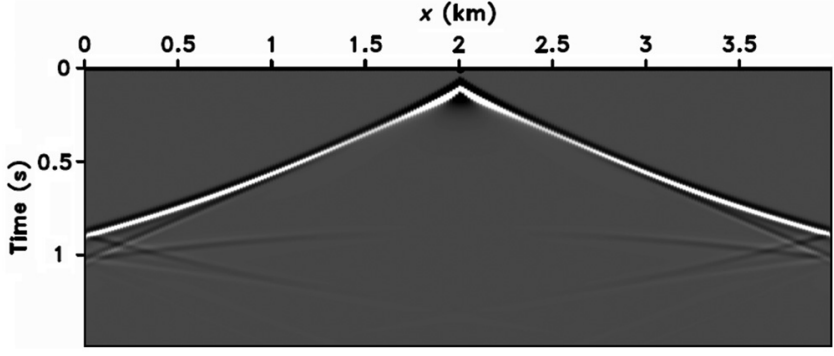

Figure 10. A common-shot gather for a source located at the surface at location $2 \mathrm{~km}$ and receivers spanning the whole surface of the domain. The velocity model is linear with velocity at the surface being $v_{0}=2 \mathrm{~km} / \mathrm{s}$ and at the gradient being $G=3 \mathrm{~s}^{-1}$. behavior of the velocity increase with depth. Such a measure can be made at many lateral points to have an effective measure of how such a gradient is changing laterally. Such information can be useful to build initial models for FWI, and specifically those applied to diving waves.

In practice, if we do not have any a priori information about $v_{0}$ and $G$, the proposed imaging moveout approximations can be applied in an alterative way. We cannot guarantee any convergence of this approach, but we believe it gives more stable estimates comparing with using imaging moveout containing both errors $\Delta v$ and $\Delta G$. The practical aspects of using imaging moveout and more sensitivity tests will be in focus for our further investigations. a)

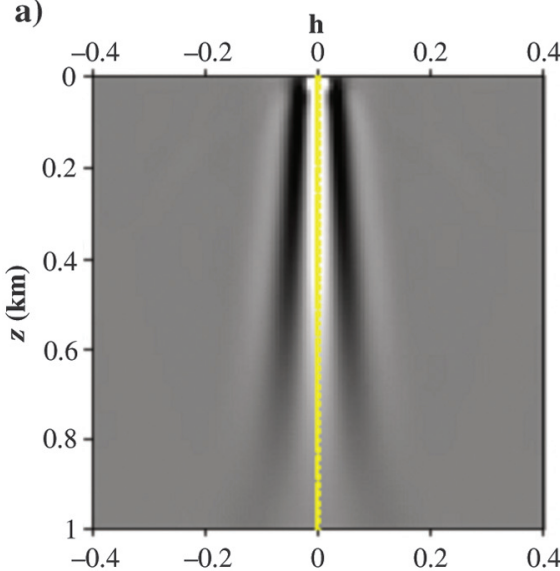

b)

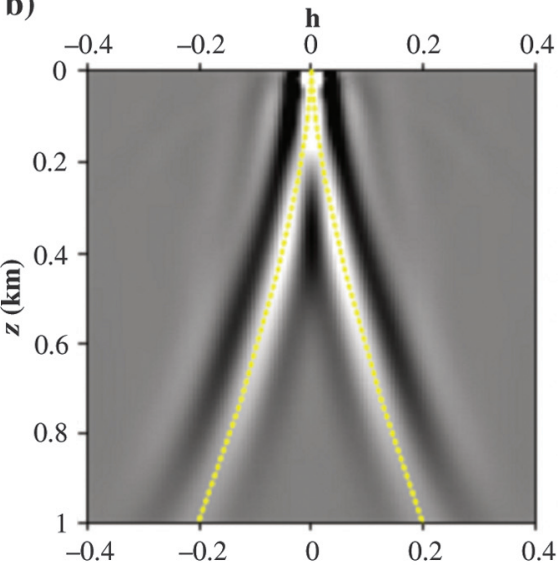

c)
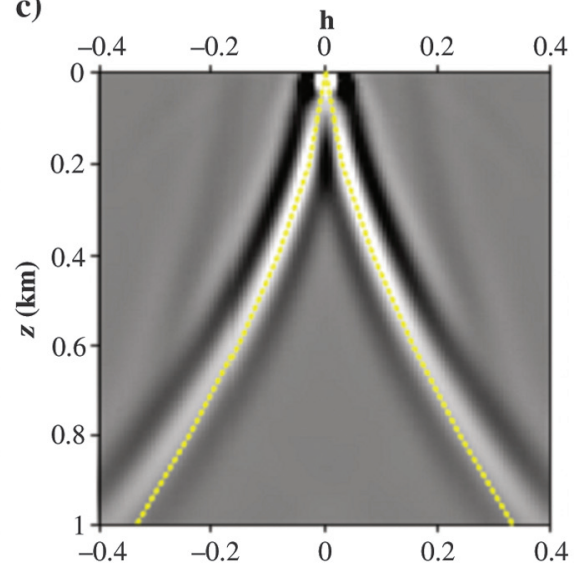

Figure 11. Common-image gathers from applying RTM on the synthetic data shown in Figure 1 (a) using the accurate velocity model with a gradient $G=3 \mathrm{~s}^{-1}$, (b) using an inaccurate gradient $G=2.5 \mathrm{~s}^{-1}$, and (c) using an inaccurate gradient $G=2 \mathrm{~s}^{-1}$. We overlay the residual curve predicted by equation 17 .

a)

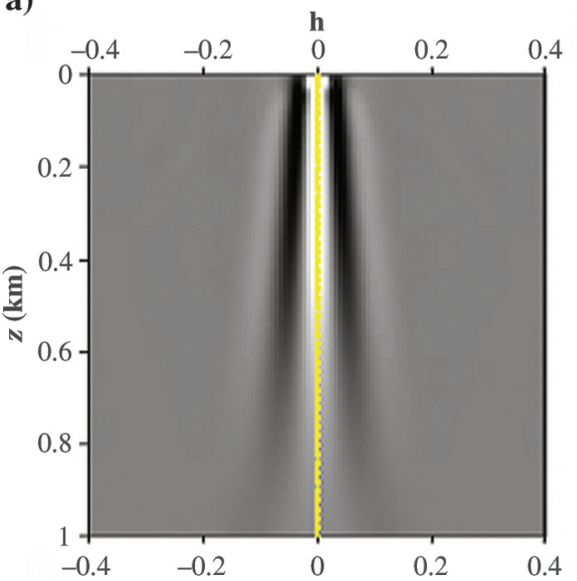

b)

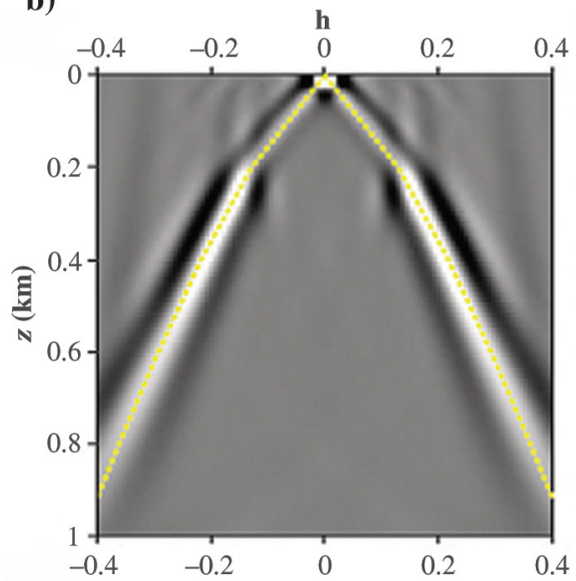

c)

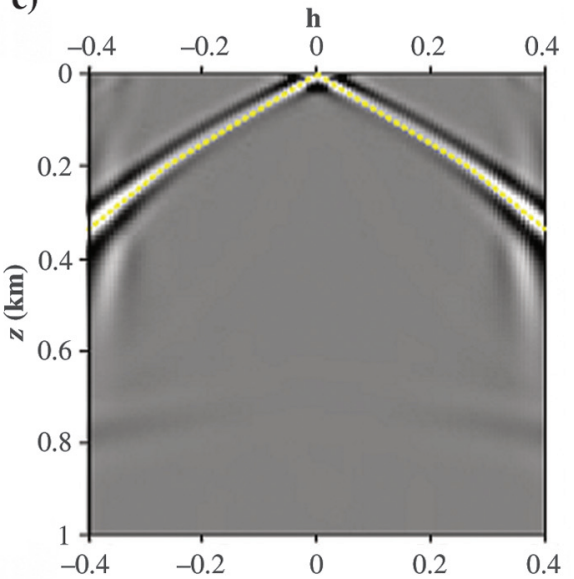

Figure 12. Common-image gathers from applying RTM on the synthetic data shown in Figure 1 (a) using the accurate velocity model with an initial velocity $v_{0}=2 \mathrm{~km} / \mathrm{s}$, (b) using an inaccurate initial velocity $v_{0}=1.5 \mathrm{~km} / \mathrm{s}$, and (c) using an inaccurate velocity $v_{0}=1 \mathrm{~km} / \mathrm{s}$. We overlay the residual curves predicted by equation 30 . 


\section{CONCLUSIONS}

We have developed formulations that describe the subsurface image gathers analytically for diving waves in a linear gradient medium. These approximations for the image point dispersal of diving waves, using the Shanks transform and rational approximations, are highly accurate especially when comparing them with those obtained from RTM. They show that the gradient and the surface velocity affect the residuals in different ways. These analytical formulations can also be utilized to scan for effective surface velocity and gradient for a starting velocity for diving-wave FWI

\section{ACKNOWLEDGMENTS}

We thank R. Plessix and M. Zuberi for useful discussions. We also acknowledge the ROSE project and KAUST for financial support.

\section{APPENDIX A}

\section{DIVING WAVE IN A MEDIUM WITH CONSTANT VELOCITY GRADIENT}

To compute the offset and traveltime equations for a diving wave in a constant velocity gradient model $v(z)=v_{0}+G z$, we take the integrals

$$
\begin{aligned}
x(p, z) & =\int_{0}^{z} \frac{p v(\xi) d \xi}{\sqrt{1-p^{2} v^{2}(\xi)}} \\
& =\frac{1}{p G}\left(\sqrt{1-p^{2} v_{0}^{2}}-\sqrt{1-p^{2} v^{2}(z)}\right)
\end{aligned}
$$

and

$$
\begin{aligned}
t(p, z) & =\int_{0}^{z} \frac{d \xi}{v(\xi) \sqrt{1-p^{2} v^{2}(\xi)}} \\
& =\frac{1}{G} \log \left(\frac{v(z)}{v_{0}} \frac{1+\sqrt{1-p^{2} v_{0}^{2}}}{1+\sqrt{1-p^{2} v^{2}(z)}}\right)
\end{aligned}
$$

The position of the turning point is defined by setting the horizontal slowness to the minimum value, $p=1 / v(z)$, in equations $\mathrm{A}-1$ and A-2:

$$
\begin{aligned}
& x_{0}(p)=\frac{\sqrt{1-p^{2} v_{0}^{2}}}{p G}, \\
& z_{0}(p)=\frac{1-p v_{0}}{p G}, \\
& t_{0}(p)=\frac{1}{G} \log \left(\frac{1+\sqrt{1-p^{2} v_{0}^{2}}}{p v_{0}}\right) .
\end{aligned}
$$

Equation A-1 can be used to trace the ray with a fixed value of horizontal slowness.

Eliminating horizontal slowness from equations for $x_{0}(p)$ and $t_{0}(p)$ in equation A-3 results in the well-known equation for diving-wave moveout in a constant velocity gradient medium:

$$
t(x)=\frac{2}{G} \log \left[\frac{G x}{2 v_{0}}+\sqrt{1+\frac{G^{2} x^{2}}{4 v_{0}^{2}}}\right]
$$

\section{APPENDIX B}

\section{TAYLOR SERIES FOR MOVEOUT CURVES IN PERTURBED MEDIA}

The moveout curve is defined as $\Delta x\left(z_{P}\right)$ in the medium with perturbation in velocity gradient by parametric equations

$$
\begin{aligned}
z_{P}(T) & =\frac{v_{0}}{(G+\Delta G)} \frac{\left(e^{G T} e^{\Delta G T}-1\right)\left(e^{G T}-e^{\Delta G T}\right)}{e^{G T}\left(e^{2 \Delta G T}+1\right)}, \\
\Delta x(T) & =x_{P}-x_{0} \\
& =\frac{v_{0}}{(G+\Delta G)} \frac{\left(e^{2 G T} e^{2 \Delta G T}-1\right)}{e^{G T}\left(e^{2 \Delta G T}+1\right)} \\
& -\frac{v_{0}}{G} \frac{\left(e^{2 G T}-1\right)}{2 e^{G T}} .
\end{aligned}
$$

Expanding $z_{P}^{3}$ and $\Delta x^{2}$ in series results in

$$
\begin{aligned}
\Delta x^{2}(T) & =\frac{\Delta G^{2}(G-\Delta G)^{2} v_{0}^{2} T^{6}}{9} \\
& +\frac{\Delta G^{2}(G-\Delta G)^{2}\left(G^{2}-4 \Delta G^{2}\right) v_{0}^{2} T^{8}}{45} \\
& +\frac{\Delta G^{2}(G-\Delta G)^{2}\left(9 G^{4}-82 G^{2} \Delta G^{2}+254 \Delta G^{4}\right) v_{0}^{2} T^{10}}{4725} \\
& +\ldots, \\
z_{P}^{3}(T) & =\frac{(G-\Delta G)^{3} v_{0}^{3} T^{6}}{8} \\
& +\frac{(G-\Delta G)^{3}\left(G^{2}-5 \Delta G^{2}\right) v_{0}^{3} T^{8}}{32} \\
& +\frac{(G-\Delta G)^{3}\left(7 G^{4}-78 G^{2} \Delta G^{2}+247 \Delta G^{4}\right) v_{0}^{3} T^{10}}{1920} \\
& +\ldots
\end{aligned}
$$

By combining series in equation B-2 together, we obtain

$$
\Delta x^{2}=a_{3} z_{P}^{3}+a_{4} z_{P}^{4}+a_{5} z_{P}^{5}+\ldots,
$$

with coefficients

$$
\begin{aligned}
& a_{3}=\frac{8}{9} \frac{\Delta G^{2}}{v_{0}(G-\Delta G)}, \\
& a_{4}=-\frac{4}{45} \frac{\Delta G^{2}\left(G^{2}-9 \Delta G^{2}\right)}{v_{0}^{2}(G-\Delta G)^{2}}, \\
& a_{5}=\frac{2}{4725} \frac{\Delta G^{2}\left(39 G^{4}-542 G^{2} \Delta G^{2}+1719 \Delta G^{4}\right)}{v_{0}^{3}(G-\Delta G)^{3}} .
\end{aligned}
$$

The moveout curve is defined as $\Delta x\left(z_{P}\right)$ in the medium with perturbation in velocity to the model top by parametric equations 


$$
\begin{aligned}
& z_{P}(p)=\frac{v_{0}}{G} \frac{\sqrt{1-r_{2}^{2}}\left(\sqrt{1-r_{1}^{2}}-1+r_{1} r_{2}\right)}{\sqrt{1-r_{1}^{2}}\left(1-r_{1} r_{2}\right)}, \\
& \Delta x(p)=\frac{v_{0}}{G} \frac{r_{1} r_{2}\left(r_{1}-r_{2}\right)}{\sqrt{1-r_{1}^{2}}\left(1-r_{1} r_{2}\right)},
\end{aligned}
$$

where $r_{1}=\sqrt{1-p^{2} v_{0}^{2}}$ and $r_{2}=\sqrt{1-p^{2}\left(v_{0}+\Delta v\right)^{2}}$.

The horizontal slowness $p_{c}$ that results in zero $z_{P}$ is different for different signs of $\Delta v$ (equation 24). Substituting horizontal slowness in equations B-5,

$$
p=\frac{p_{c}(1-s)}{v_{0}},
$$

and expanding $\Delta x(s)$ and $z_{P}(s)$ in series

$$
\begin{aligned}
\Delta x(s) & =u_{0}+u_{1} s+u_{2} s^{2}+\ldots, \\
z_{P}(s) & =w_{0}+w_{1} s+w_{2} s^{2}+\ldots,
\end{aligned}
$$

where the coefficients $u_{j}, \omega_{j}, j=0,1,2$, are algebraically complicated functions of $p_{c}$, we can obtain

$$
\Delta x(z)=\Delta x(0)+a_{1} z_{P}+a_{2} z_{P}^{2}
$$

with coefficients

$$
\begin{aligned}
& a_{1}=\frac{\sqrt{1-p_{c}^{2} v_{0}^{2}}-\sqrt{1-p_{c}^{2} v_{0}^{2}(1+\gamma)^{2}}}{p_{c}^{2} v_{0}^{2}(1+\gamma)}, \\
& a_{2}=\frac{G\left(\sqrt{1-p_{c}^{2} v_{0}^{2}}-\sqrt{1-p_{c}^{2} v_{0}^{2}(1+\gamma)^{2}}\right)\left(1-\sqrt{\left(1-p_{c}^{2} v_{0}^{2}\right)\left(1-p_{c}^{2} v_{0}^{2}(1+\gamma)^{2}\right)}\right)^{3}}{2 v_{0}^{4} p_{c}^{3}(1+\gamma)^{2}\left(\sqrt{\left(1-p_{c}^{2} v_{0}^{2}\right)\left(1-p_{c}^{2} v_{0}^{2}(1+\gamma)^{2}\right)}-1+p_{c}^{4} v_{0}^{4}(1+\gamma)^{2}\right)},
\end{aligned}
$$

and $\Delta x(0)$ given in equation 25.

\section{REFERENCES}

Levin, F., 1996, Anatomy of diving waves: Geophysics, 61, 1417-1424, doi: $10.1190 / 1.1444066$.

Plessix, R.-E., and Q. Cao, 2011, A parametrization study for surface seismic full waveform inversion in an acoustic vertical transversely isotropic medium: Geophysical Journal International, 185, 539-556, doi: 10.1111/j .1365-246X.2011.04957.x.

Plessix, R.-E., and H. Rynja, 2010, VTI full waveform inversion: A parameterization study with a narrow azimuth streamer data example: 80th Annual International Meeting, SEG, Expanded Abstracts, 962-966.

Pratt, R. G., Z.-M. Song, P. Williamson, and M. Warner, 1996, Two-dimensional velocity models from wide-angle seismic data by wavefield inversion: Geophysical Journal International, 124, 323-340, doi: 10.1111/j .1365-246X.1996.tb07023.x.

Shen, P., 2013, Subsurface focusing measurement of diving waves and its application to reflection tomography: 75th Annual International Conference and Exhibition, EAGE, Extended Abstracts, Th1005.

Sirgue, L., and R. Pratt, 2004, Efficient waveform inversion and imaging: A strategy for selecting temporal frequencies: Geophysics, 69, 231-248, doi: $10.1190 / 1.1649391$

Slotnick, M., 1959, Lessons in seismic computing: SEG.

Tang, Y., S. Lee, A. Baumstein, and D. Hinkley, 2013, Tomographically enhanced full wavefield inversion: 83rd Annual International Meeting, SEG, Expanded Abstracts, 1037-1041.

Virieux, J., and S. Operto, 2009, An overview of full-waveform inversion in exploration geophysics: Geophysics, 74, no. 6, WCC1-WCC26, doi: 10 $.1190 / 1.3238367$. 